\title{
Flow Dynamics in Restricted Geometries: A Mathematical Concept Based on Bloch NMR Flow Equation and Boubaker Polynomial Expansion Scheme
}

\author{
Omotayo Bamidele Awojoyogbe ${ }^{*}$, Oluwaseun Michael Dada ${ }^{1}$, Karem Boubaker ${ }^{2}$, \\ Omoniyi Adewale Adesola ${ }^{1}$ \\ ${ }^{1}$ Department of Physics, Federal University of Technology, Minna, Nigeria \\ ${ }^{2}$ UPDS/ESSTT/63 Rue Sidi Jabeur 5100, Mahdia, Tunisia \\ Email: " awojoyogbe@yahoo.com
}

Received August 4, 2013; revised September 25, 2013; accepted October 2, 2013

Copyright (C) 2013 Omotayo Bamidele Awojoyogbe et al. This is an open access article distributed under the Creative Commons Attribution License, which permits unrestricted use, distribution, and reproduction in any medium, provided the original work is properly cited.

\begin{abstract}
Computational techniques are invaluable to the continued success and development of Magnetic Resonance Imaging (MRI) and to its widespread applications. New processing methods are essential for addressing issues at each stage of MRI techniques. In this study, we present new sets of non-exponential generating functions representing the NMR transverse magnetizations and signals which are mathematically designed based on the theory and dynamics of the Bloch NMR flow equations. These signals are functions of many spinning nuclei of materials and can be used to obtain information observed in all flow systems. The Bloch NMR flow equations are solved using the Boubaker polynomial expansion scheme (BPES) and analytically connect most of the experimentally valuable NMR parameters in a simplified way for general analyses of magnetic resonance imaging with adiabatic condition.
\end{abstract}

Keywords: Bloch NMR Flow Equations; Boubaker Polynomial Expansion Scheme (BPES); Magnetic Resonance Imaging (MRI); Adiabatic Condition

\section{Introduction}

Flow through porous media represents a vast field of study with many scientific and engineering applications [1-7]. A great number of experimental and theoretical studies on flow in restricted motion using NMR are available in the literature [1-18]. Most of these studies are based on either numerical or approximation solutions of Bloch NMR equations. However, it will be fundamental and ideal if the theoretical and experimental application of MRI for flow analysis in restricted geometry is based on the analytical solutions of Bloch NMR equations. This has been claimed over the years to be the best approach for obtaining fundamental information to accurately access fluid dynamical properties in porous media/restricted geometry. It is possible to derive necessary relationships analytically for free motion. However, in the case of restricted motion for which porous media are defined, the macroscopic approach becomes mathematically intracta-

"Corresponding author. ble. Thus, in general case, one is forced to use different method to find mathematical relation for the MRI signal in terms of NMR experimental parameters [19-21].

In this investigation, we solved the Bloch NMR flow equation which is transformable to Bessel equation of order zero using the Boubaker Polynomial Expansion Scheme to obtain the NMR transverse magnetization for the analysis of flow in anisotropic fluid flow. The relationships between fluid velocity, the NMR relaxation rates and the path length $x$ for cerebrospinal fluid, white and gray matter of human cerebrum are demonstrated. The Boubaker Polynomials Expansion Scheme BPES is a resolution protocol which has been successfully applied to several applied-physics and mathematics problems. Solutions have been proposed through the BPES in many fields such as numerical analysis [22-27], theoretical physics [24-29], mathematical algorithms [26], heat transfer [30,31], homodynamics [28,29], material characterization [32], fuzzy systems modeling [31] and boilogy $[32,33]$. 


\section{Mathematical Analysis}

The BPES protocol ensures the validity of the related boundary conditions regardless of the main features of the equation. The BPES is mainly based on Boubaker polynomials first derivatives properties

$$
\left\{\begin{array}{l}
\left.\sum_{q=1}^{N} B_{4 q}(x)\right|_{x=0}=-2 N \neq 0 \\
\left.\sum_{q=1}^{N} B_{4 q}(x)\right|_{x=r_{q}}=0
\end{array}\right.
$$

and

$$
\left\{\begin{array}{l}
\left.\sum_{q=1}^{N} \frac{\mathrm{d} B_{4 q}(x)}{\mathrm{d} x}\right|_{x=0}=0 \\
\left.\sum_{q=1}^{N} \frac{\mathrm{d} B_{4 q}(x)}{\mathrm{d} x}\right|_{x=r_{q}}=\sum_{q=1}^{N} H_{q} \\
\text { with: } H_{n}=B_{4 n}^{\prime}\left(r_{n}\right)=\left(\frac{4 r_{n}\left[2-r_{n}^{2}\right] \times \sum_{q=1}^{n} B_{4 q}^{2}\left(r_{n}\right)}{B_{4(n+1)}\left(r_{n}\right)}+4 r_{n}^{3}\right)
\end{array}\right.
$$

In this investigation, the Boubaker Polynomials Expansion Scheme BPES has been applied to the boundary-valued second order Bloch NMR flow differential equation through setting the expression:

$$
M_{y}(x)=\frac{1}{2 N_{0}} \sum_{k=1}^{N_{0}} \lambda_{k} \times B_{4 k}\left(x r_{k}\right)
$$

where $M_{y}(x)$ are the time independent NMR transverse magnetizations, $B_{4 k}$ are the $4 k$-order Boubaker polynomials, $x \in[0,1]$ is the normalized variable, $r_{k}$ are $B_{4 k}$ minimal positive roots, $N_{0}$ is a prefixed integer and $\left.\lambda_{k}\right|_{k=1, \cdots, N_{0}}$ are unknown pondering real coefficients.

Based on the conditions which may conform to the real-time experimental arrangements, we obtained a second order non homogeneous differential equation from the Bloch NMR flow equation [34,35] at Larmor frequency:

$$
f_{o}=\gamma B-\omega=0
$$

The $x, y, z$ components (in the rotating frame) of the magnetization of a fluid moving with spatially varying velocity $v$ is given by the Bloch equations which may be written as follows:

$$
\frac{\mathrm{d} M_{x}}{\mathrm{~d} t}=v \cdot \operatorname{grad} M_{x}+\frac{\partial M_{x}}{\partial t}=-\frac{M_{x}}{T_{2}}
$$

$$
\begin{gathered}
\frac{\mathrm{d} M_{y}}{\mathrm{~d} t}=v \cdot \operatorname{grad} M_{y}+\frac{\partial M_{y}}{\partial t}=\gamma M_{z} B_{1}(x)-\frac{M_{y}}{T_{2}} \\
\frac{\mathrm{d} M_{z}}{\mathrm{~d} t}=v \cdot \operatorname{grad} M_{z}+\frac{\partial M_{z}}{\partial t}=-\gamma M_{y} B_{1}(x)+\frac{\left(M_{0}-M_{z}\right)}{T_{1}}
\end{gathered}
$$

Subject to the following conditions:

1) $M_{\mathrm{o}} \neq M_{z}$ a situation which holds well in general and in particular when the RF $B_{1}(x)$ field is strong say of the order of $1.0 \mathrm{G}$ or more.

2) Before entering signal detector coil, fluid particles has magnetization.

$M_{x}=0, M_{y}=0$.

3) If $B_{1}(x)$ is large; $B_{1}(x) \gg 1 \mathrm{G}$ or more so that $M_{y}$ of the fluid bolus changes appreciably from the equilibrium magnetization $M_{o}$.

$\gamma$ denotes the gyromagnetic ratio of fluid spins; $\omega / 2 \pi$ is the RF excitation frequency; $f_{0} / \gamma$ is the off-resonance field in the rotating frame of reference. $T_{1}$ and $T_{2}$ are the spin-lattice and spin-spin relaxation times respectively, the reciprocals of $T_{1}$ and $T_{2}$ are defined as relaxation rates. $M_{o}$ is the equilibrium magnetization and RF $B_{1}$ is the spatially varying magnetic field [35] which may be designed as

$$
\gamma B_{1}(x)=\gamma g x
$$

where $g$ is the field gradient. Equations (5) and (6) give a second order non-homogenous differential equation called the Bloch NMR flow equation:

$$
\frac{\mathrm{d}^{2} M_{y}}{\mathrm{~d} x^{2}}+\frac{T_{0}}{v} \frac{\mathrm{d} M_{y}}{\mathrm{~d} x}+\frac{S(x)}{v^{2}} M_{y}=\frac{M_{0}}{v^{2} T_{1}} \gamma B_{1}(x)
$$

where $T_{0}=\frac{1}{T_{1}}+\frac{1}{T_{2}}, \quad S(x)=\gamma^{2} B_{1}^{2}(x)+\frac{1}{T_{1} T_{2}}$

In NMR systems, when the RF $B_{1}$ field is applied, $M_{y}$ has a maximum value when RF $B_{1}$ has maximum amplitude and $M_{o} \approx 0$. In biological systems especially at the molecular level we need to solve Equation (8) to provide velocity profiles for different tissues materials such that

$$
\begin{gathered}
x=\frac{\alpha v}{T_{o}}=v n T R \\
n=\frac{T_{o}(T R)}{\alpha}
\end{gathered}
$$

where $n$ is the number of pulses, $T R$ is the repetition time. If $\tau$ is the time between two pulses, we write:

$$
\tau=\frac{T R}{n}=\frac{\alpha}{T_{0}}
$$

For adiabatic condition, Equation (8) becomes: 


$$
x \frac{\mathrm{d}^{2} M_{y}}{\mathrm{~d} x^{2}}+\alpha \frac{\mathrm{d} M_{y}}{\mathrm{~d} x}+\gamma^{2}\left(\frac{\alpha}{T_{o}}\right)^{2} g^{2} x M_{y}=0
$$

where

$$
\gamma^{2} B_{1}^{2}(x) \gg \frac{1}{T_{1} T_{2}}
$$

Equations (13) and (14) can be solved using the Boubaker polynomial expansion scheme [21-23] with boundary conditions based on traditional NMR procedures.

$$
\left\{\begin{array}{l}
x \frac{\mathrm{d}^{2} M_{y}}{\mathrm{~d} x^{2}}+\alpha \frac{\mathrm{d} M_{y}}{\mathrm{~d} x}+\beta x M_{y}^{n}=0 \\
M_{y}(0)=1 ; \frac{\mathrm{d} M_{y}(0)}{\mathrm{d} x}=0
\end{array}\right.
$$

where $\beta$ is a constant which is unique to the NMR system being described. For this system, the gradient field is chosen (under the condition $\alpha=2$ ) such that

$$
g=\frac{T_{o}}{2 \gamma}
$$

We define $n$ as a dimensionless variable

$$
f=\frac{n \rho \alpha}{T_{o} x^{2}}
$$

where $\rho$ is a special flow property of the fluid (for this analysis $\rho \ll 1), \alpha$ is dimenssioless constant and $f$ is a property of the medium. Equations (13) and (14) reduce to:

$$
\begin{aligned}
& \frac{1}{2 N_{0}} \sum_{k=1}^{N_{0}} \lambda_{k, n} \times \frac{\mathrm{d}^{2} B_{4 k}\left(x r_{k}\right)}{\mathrm{d} x^{2}}+\frac{1}{x N_{0}} \sum_{k=1}^{N_{0}} \lambda_{k, n} \times \frac{\mathrm{d} B_{4 k}\left(x r_{k}\right)}{\mathrm{d} x} \\
& =\left(-\frac{1}{N_{0}} \sum_{k=1}^{N_{0}} \lambda_{k, n} \times B_{4 k}\left(x r_{k}\right)\right)^{2}
\end{aligned}
$$

The BPES solution is obtained by determining the non-null set of coefficients $\left.\tilde{\lambda}_{k}\right|_{k=1, \cdots, N_{0}}$ that minimizes the absolute difference $\Delta_{N_{0}}$ :

$$
\Delta_{N_{0}}=\left|\left(\frac{1}{2 N_{0}} \sum_{k=1}^{N_{0}} \tilde{\lambda}_{k, n} \times \Lambda_{k}\right)-\left(\frac{1}{2 N_{0}} \sum_{k=1}^{N_{0}} \tilde{\lambda}_{k, n} \times \Lambda_{k}^{\prime}\right)\right|
$$

with:

$$
\begin{aligned}
& \Lambda_{k}=r_{k}^{2} \int_{0}^{1} \frac{\mathrm{d}^{2} B_{4 k}}{\mathrm{~d} x^{2}}\left(x \times r_{k}\right) \mathrm{d} x \\
& \Lambda_{k}^{\prime}=-r_{k} \int_{0}^{1}\left(\frac{2}{x} \times \frac{\mathrm{d} B_{4 k}}{\mathrm{~d} x}\left(x \times r_{k}\right)-\left(-\frac{1}{N_{0}} \sum_{k=1}^{N_{0}} B_{4 k}\left(x \times r_{k}\right)\right)^{2}\right) \mathrm{d} x
\end{aligned}
$$

The final solution is:

$$
\left.M_{y, n}(x)\right|_{n=2,3,4,5}=\frac{1}{2 N_{0}} \sum_{k=1}^{N_{0}} \tilde{\lambda}_{k, n} \times B_{4 k}\left(x r_{k}\right)
$$

\section{Analysis of Results}

From Equations (7), (9)-(13), we obtain for the value of $\alpha$ $=2$, the following

$$
\begin{gathered}
x=\frac{2 v}{T_{o}} \\
f=\frac{n \rho \alpha}{T_{o} x^{2}}=\frac{n \rho T_{o}}{2 v^{2}}
\end{gathered}
$$

Tables 1-4 show how the fluid velocity and relaxation parameters changes with $x$ for different human tissues materials at $1.5 \mathrm{~T}$.

The tables show the usefulness of BPES to different tissues on MRI scan. They can also be used to observe the same tissue materials at different locations.

Figure 1 shows the NMR transverse magnetization when the value of $x$ is small, high and very high for the Boubaker polynomial expansion scheme (BPES). The number of pulses $n$ have more influence on the NMR signal when the value of $x$ is small that when it is high. This can be useful to determine the number of pulses needed for a particular NMR experiment.

Figures $\mathbf{2}$ and $\mathbf{3}$ show velocity profiles for different tissues materials. The color bands represent the different magnitude of the fluid velocity for different tissue. For example in Figure 2, the velocity profile is $0.030 \mathrm{~m} / \mathrm{s}$ in cerebrospinal fluid while it is $0.25 \mathrm{~m} / \mathrm{s}$ in gray matter for the same color band with the $T_{1}$ and $T_{2}$ relaxation rates providing tissue contrast.

Table 1. Values of the path length, velocity and the relaxation rate for cerebrospinal fluid at $1.5 \mathrm{~T}$.

\begin{tabular}{ccccc}
\hline \multicolumn{5}{c}{ Cerebrospinal Fluid } \\
\hline$x$ & $x^{2}$ & $\tau=\alpha / T_{0}$ & $T_{0}$ & $v$ \\
\hline 0.046114 & 0.002127 & 0.332266 & 6.019278 & 0.138786 \\
0.044374 & 0.001969 & 0.307664 & 6.500591 & 0.144229 \\
0.042634 & 0.001818 & 0.284009 & 7.042029 & 0.150115 \\
0.040894 & 0.001672 & 0.261300 & 7.654041 & 0.156502 \\
0.039154 & 0.001533 & 0.239537 & 8.349447 & 0.163457 \\
0.037414 & 0.001400 & 0.218720 & 9.144115 & 0.171059 \\
0.035674 & 0.001273 & 0.198849 & 10.05788 & 0.179402 \\
0.033934 & 0.001152 & 0.179924 & 11.11578 & 0.188601 \\
0.032194 & 0.001036 & 0.161946 & 12.34980 & 0.198795 \\
0.030454 & 0.000927 & 0.144913 & 13.80134 & 0.210153 \\
0.028714 & 0.000824 & 0.128827 & 15.52468 & 0.222888 \\
0.026974 & 0.000728 & 0.113687 & 17.59216 & 0.237266 \\
0.025234 & 0.000637 & 0.099493 & 20.10193 & 0.253626 \\
0.023494 & 0.000552 & 0.086245 & 23.18975 & 0.272410 \\
0.021754 & 0.000473 & 0.073943 & 27.04779 & 0.294199 \\
0.000000 & 0.000000 & 0.000000 & $\infty$ & $\infty$ \\
\hline & & & & \\
\hline
\end{tabular}


Table 2. Values of the path length, velocity and the relaxation rate for gray matter of the cerebrum at $1.5 \mathrm{~T}$.

\begin{tabular}{|c|c|c|c|c|}
\hline \multicolumn{5}{|c|}{ Gray Matter } \\
\hline$x$ & $x^{2}$ & $\tau=\alpha / T_{0}$ & $T_{0}$ & $v$ \\
\hline 0.046114 & 0.002127 & 1.329063 & 1.504819 & 0.034697 \\
\hline 0.044374 & 0.001969 & 1.230657 & 1.625148 & 0.036057 \\
\hline 0.042634 & 0.001818 & 1.136036 & 1.760507 & 0.037529 \\
\hline 0.040894 & 0.001672 & 1.045200 & 1.913510 & 0.039126 \\
\hline 0.039154 & 0.001533 & 0.958147 & 2.087362 & 0.040864 \\
\hline 0.037414 & 0.001400 & 0.874880 & 2.286029 & 0.042765 \\
\hline 0.035674 & 0.001273 & 0.795396 & 2.514469 & 0.044851 \\
\hline 0.033934 & 0.001152 & 0.719698 & 2.778944 & 0.047150 \\
\hline 0.032194 & 0.001036 & 0.647784 & 3.087451 & 0.049699 \\
\hline 0.030454 & 0.000927 & 0.579654 & 3.450335 & 0.052538 \\
\hline 0.028714 & 0.000824 & 0.515309 & 3.881169 & 0.055722 \\
\hline 0.026974 & 0.000728 & 0.454748 & 4.398041 & 0.059316 \\
\hline 0.025234 & 0.000637 & 0.397972 & 5.025483 & 0.063407 \\
\hline 0.023494 & 0.000552 & 0.344980 & 5.797437 & 0.068102 \\
\hline 0.021754 & 0.000473 & 0.295773 & 6.761946 & 0.159695 \\
\hline 0.000000 & 0.000000 & 0.000000 & $\infty$ & $\infty$ \\
\hline
\end{tabular}

Table 3. Values of the path length, velocity and the relaxation rate for white matter of the cerebrum at $1.5 \mathrm{~T}$.

\begin{tabular}{ccccc}
\hline \multicolumn{5}{c}{ White Matter } \\
\hline$x$ & $x^{2}$ & $\tau=\alpha / T_{0}$ & $T_{0}$ & $v$ \\
\hline 0.046114 & 0.002127 & 1.772084 & 1.128615 & 0.026022 \\
0.044374 & 0.001969 & 1.640877 & 1.218861 & 0.027043 \\
0.042634 & 0.001818 & 1.514715 & 1.320380 & 0.028147 \\
0.040894 & 0.001672 & 1.393599 & 1.435133 & 0.029344 \\
0.039154 & 0.001533 & 1.277530 & 1.565521 & 0.030648 \\
0.037414 & 0.001400 & 1.166506 & 1.714522 & 0.032074 \\
0.035674 & 0.001273 & 1.060529 & 1.885852 & 0.033638 \\
0.033934 & 0.001152 & 0.959597 & 2.084208 & 0.035363 \\
0.032194 & 0.001036 & 0.863711 & 2.315588 & 0.037274 \\
0.030454 & 0.000927 & 0.772872 & 2.587751 & 0.039404 \\
0.028714 & 0.000824 & 0.687078 & 2.910877 & 0.041791 \\
0.026974 & 0.000728 & 0.606331 & 3.298531 & 0.044487 \\
0.025234 & 0.000637 & 0.530629 & 3.769112 & 0.047555 \\
0.023494 & 0.000552 & 0.459973 & 4.348078 & 0.051077 \\
0.021754 & 0.000473 & 0.394364 & 5.07146 & 0.055162 \\
0.000000 & 0.000000 & 0.000000 & $\infty$ & $\infty$ \\
\hline
\end{tabular}

Table 4. Values of the path length, velocity and the relaxation rate for white matter of cystic tumor at $1.5 \mathrm{~T}$.

\begin{tabular}{ccccc}
\hline \multicolumn{5}{c}{ Cystic tumor } \\
\hline$x$ & $x^{2}$ & $\tau=\alpha / T_{0}$ & $T_{0}$ & $v$ \\
\hline 0.046114 & 0.002127 & 0.189866 & 10.53374 & 0.242876 \\
0.044374 & 0.001969 & 0.175808 & 11.37603 & 0.252400 \\
0.042634 & 0.001818 & 0.162291 & 12.32355 & 0.262701 \\
0.040894 & 0.001672 & 0.149314 & 13.39457 & 0.273879 \\
0.039154 & 0.001533 & 0.136878 & 14.61153 & 0.286050 \\
0.037414 & 0.001400 & 0.124983 & 16.0022 & 0.299353 \\
0.035674 & 0.001273 & 0.113628 & 17.60129 & 0.313954 \\
0.033934 & 0.001152 & 0.102814 & 19.45261 & 0.330052 \\
0.032194 & 0.001036 & 0.092541 & 21.61216 & 0.347891 \\
0.030454 & 0.000927 & 0.082808 & 24.15235 & 0.367768 \\
0.028714 & 0.000824 & 0.073616 & 27.16819 & 0.390054 \\
0.026974 & 0.000728 & 0.064964 & 30.78629 & 0.415215 \\
0.025234 & 0.000637 & 0.056853 & 35.17838 & 0.443846 \\
0.023494 & 0.000552 & 0.049283 & 40.58206 & 0.476717 \\
0.021754 & 0.000473 & 0.042253 & 47.33363 & 0.514848 \\
0.000000 & 0.000000 & 0.000000 & $\infty$ & $\infty$ \\
\hline
\end{tabular}

Based on Equation (19) and Tables 1-4, the analysis of fluid velocity, relaxation rates and the path length $x$ can be described within the following three limits:

$$
\begin{aligned}
& f=\frac{n \alpha \rho}{T_{o} x^{2}} \ll 1 \\
& f=\frac{n \alpha \rho}{T_{o} x^{2}}=1 \\
& f=\frac{n \alpha \rho}{T_{o} x^{2}} \gg 1
\end{aligned}
$$

Equation (21) is the short time limit where the particle does not flow far enough during time $n \frac{\alpha}{T_{o}}$ to feel the effect of parameter $\rho$. When $f \approx 1$, as shown in equation (22) some of the particles feel the effects of restriction and the value of $\rho$ measured within this time scale will be a function of $n \frac{\alpha}{T_{o}}$. Equation (23) shows that the time is long enough for all the particles to feel the effects of restriction and the displacement of the particle depends not on time $n \frac{\alpha}{T_{o}}$, but only on path length $x$. This indi- 

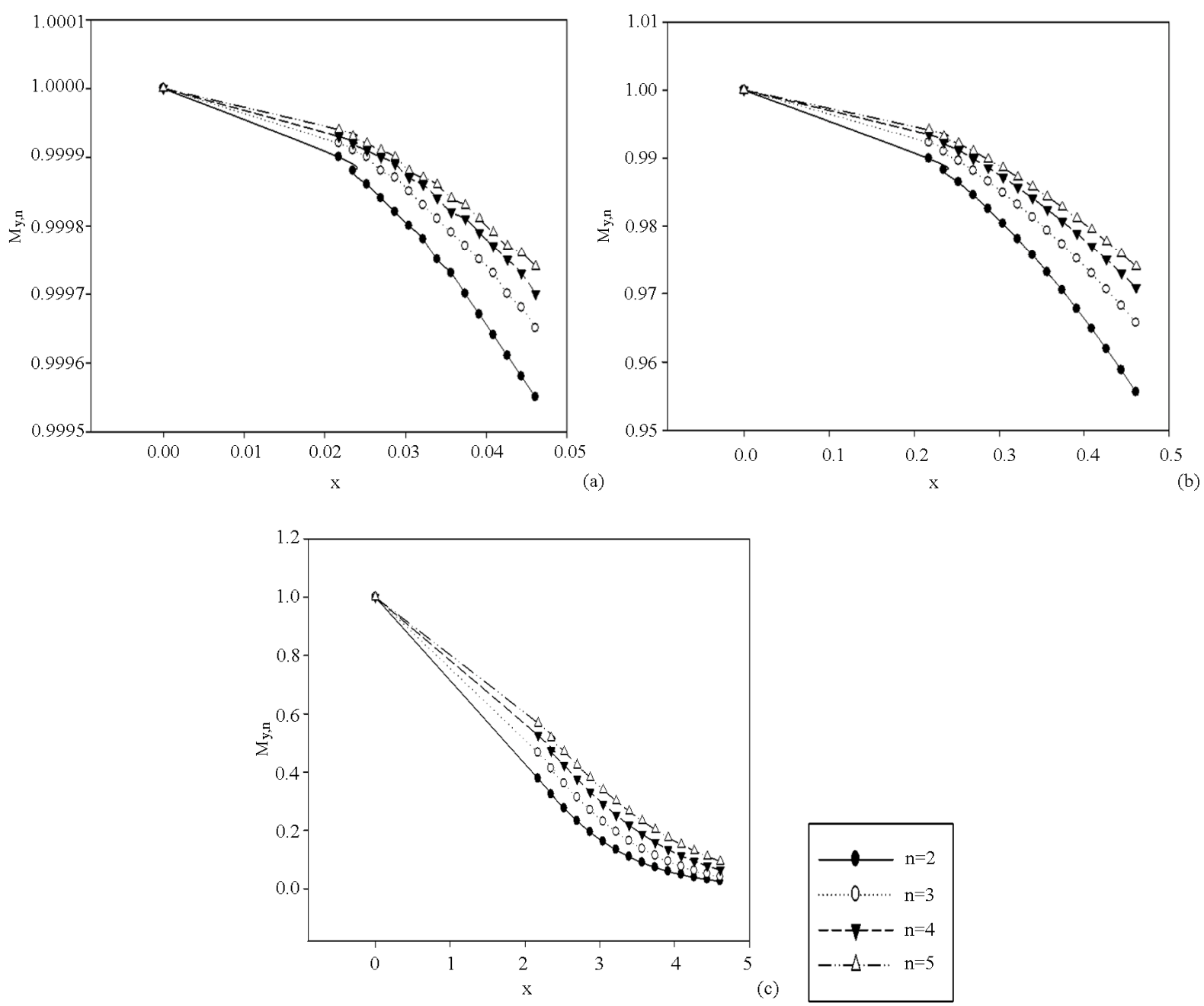

Figure 1. Plots of the NMR transverse magnetization against (a) Small values of $x$; (b) Higher values of $x$; (c) Much higher values of $x$ for the Boubaker polynomial expansion scheme (BPES).

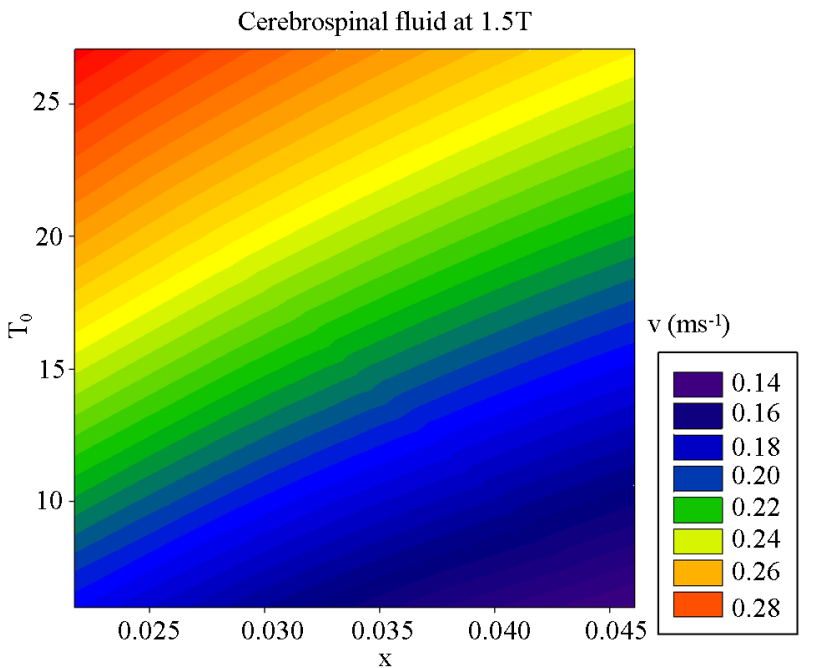

(a)

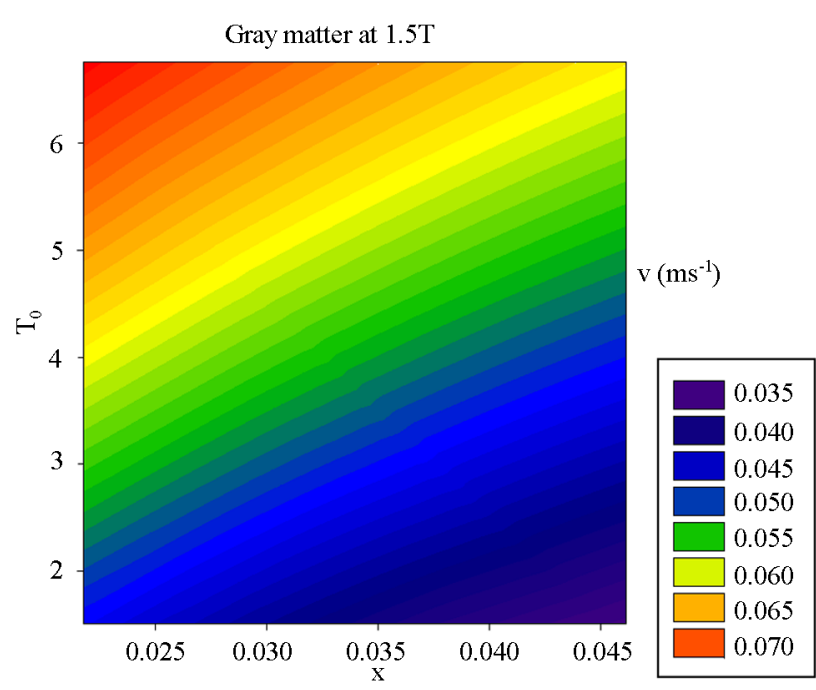

(b)

Figure 2. Plots of the fluid velocity against the relaxation rate and the path length $x$ for cerebrospinal fluid and gray matter within the human brain at a static magnetic field of $1.5 \mathrm{~T}$. 


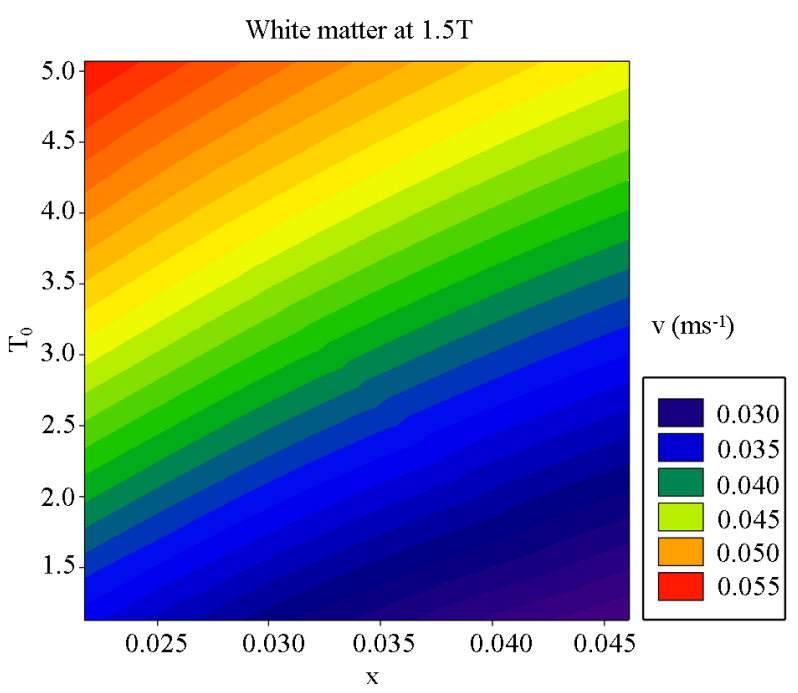

(a)

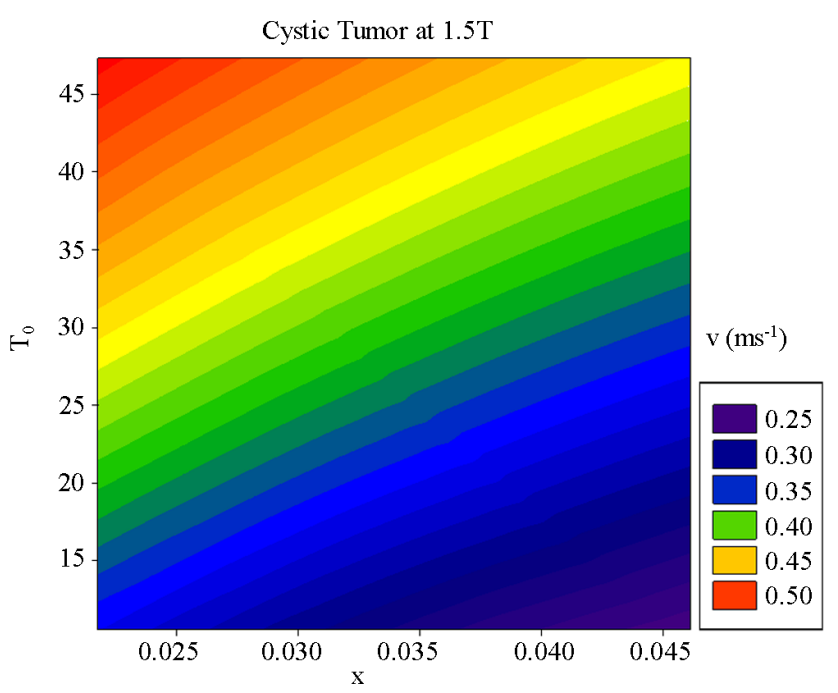

(b)

Figure 3. Plots of the fluid velocity against the relaxation rate and the path length $x$ for white matter of human cerebrum and cystic tumor at a static magnetic field of $1.5 \mathrm{~T}$.

cates that the value of $n$ and not $\rho$, in Equations (13) and (14) as solved by the Boubaker polynomial expansion scheme is very significant for the analysis of flow in restricted geometry where the measured fluid velocity depends of the relaxation parameters as shown in Figures 2 and 3. We may conclude that $f$, has a memory of the chemical differences within the spin's immediate environment or the magnitude of the static magnetic field $B_{o}$. Therefore, selecting a particular value of $x$ may correspond to selecting certain magnitude of $B_{o}$ field or the molecular imprints of the tissue containing a flowing spin. The values of $\rho$ used in this study, is for computational purposes.

\section{Conclusion}

A mathematical concept of magnetic resonance imaging for flow analyses in restricted geometries has been presented by solving the Bloch flow equation using the Boubaker polynomial expansion scheme (BPES). These demonstrate the usefulness of Bloch NMR flow equation and the Boubaker polynomial expansion scheme for studying fluid flow in restricted geometries to obtain the NMR transverse magnetization for the analyses of flow in anisotropic fluid flow. The relationship between fluid velocity, the NMR relaxation rates and the path length $x$ for cerebrospinal fluid, white and gray matter of human cerebrum as demonstrated provides tissue contrast for different tissues materials. This can prove to be a very good starting point for building more sensitive and less expensive magnetic resonance imaging sequences.

\section{Acknowledgements}

The authors acknowledge the support from Federal Uni- versity of Technology, Minna, Nigeria through the STEP $\mathrm{B}$ research programme of the World Bank.

\section{REFERENCES}

[1] S. Ogawa, T. M. Lee, A. S. Nayak and P. Glynn, "Oxygenation-Sensitive Contrast in Magnetic Resonance Image of Rodent Brain at High Magnetic Fields," Magnetic Resonance in Medicine, Vol. 14, No. 1, 1990, pp. 68-78. http://dx.doi.org/10.1002/mrm.1910140108

[2] J. J. Dejerine, "Anatomie des Centres Nerveux," Rueff, Paris, 1895.

[3] W. J. S. Krieg, "Connections of the Cerebral Cortex," Brain Books, Evanston, 1963.

[4] W. J. S. Krieg, "Architectonics of Human Cerebral Fiber Systems," Brain Books, Evanston, 1973.

[5] K. Pribam and P. MacLean, "Neuronographic Analysis of Medial and Basal Cerebral Cortex," Journal of Neurophysiology, Vol. 16, No. 3, 1953, pp. 324-340.

[6] D. G. Whitlock and W. J. H. Nauta, "Subcortical Projections from Temporal Neocortex in Macaca Mulatto," Journal of Comparative Neurology, Vol. 106, No. 1, 1956, pp. 183-212. http://dx.doi.org/10.1002/cne.901060107

[7] B. H. Turner, M. Mishkin and M. Knapp, "Organization of the Amygdalopetal Projections from Modality-Specific Cortical Association Areas in the Monkey," Journal of Comparative Neurology, Vol. 191, No. 4, 1980, pp. 515543. http://dx.doi.org/10.1002/cne.901910402

[8] A. Yagishita, I. Nakano, M. Oda and A. Hirano, "Location of the Corticospinal Tract in the Internal Capsule at MR Imaging," Radiology, Vol. 191, No. 2, 1994, pp. 455460.

[9] P. Godement, J. Vanselow, S. Thanos and F. Bonhoeffer, "A Study in Developing Visual Systems with a New Method of Staining Neurones and Their Processes in Fixed 
Tissue," Development, Vol. 101, No. 4, 1987, pp. 697713.

[10] S. Mori, B. J. Crain, V. P. Chacko and P. C. van Zijl, "Three-Dimensional Tracking of Axonal Projections in the Brain by Magnetic Resonance Imaging," Annals of Neurology, Vol. 45, No. 2, 1999, pp. 265-269.

http://dx.doi.org/10.1002/1531-8249(199902)45:2<265:: AID-ANA21 $>3.0 . \mathrm{CO} ; 2-3$

[11] T. E. Conturo, N. F. Lori, T. S. Cull, E. Akbudak, A. Z. Snyder, J. S. Shimony, et al., "Tracking Neuronal Fiber Pathways in the Living Human Brain," Proceedings of the National Academy Sciences of the USA, Vol. 96, No. 18, 1999, pp. 10422-10427.

http://dx.doi.org/10.1073/pnas.96.18.10422

[12] P. J. Basser, S. Pajevic, C. Pierpaoli, J. Duda and A. Aldroubi, "In Vivo Fiber Tractography Using DT-MRI Data," Magnetic Resonance in Medicine, Vol. 44, No. 4, 2000, pp. 625-632.

http://dx.doi.org/10.1002/1522-2594(200010)44:4<625:: AID-MRM17>3.0.CO;2-O

[13] C. R. Tench, P. S. Morgan, M. Wilson and L. D. Blumhardt, "White Matter Mapping Using Diffusion Tensor MRI," Magnetic Resonance in Medicine, Vol. 47, No. 5, 2002, pp. 967-972. http://dx.doi.org/10.1002/mrm.10144

[14] C. R. Tench, P. S. Morgan, L. D. Blumhardt and C. Constantinescu, "Improved White Matter Fiber Tracking Using Stochastic Labeling," Magnetic Resonance in Medicine, Vol. 48, No. 4, 2002, pp. 677-683. http://dx.doi.org/10.1002/mrm.10266

[15] M. A. Koch, D. G. Norris and M. Hund-Georgiadis, "An Investigation of Functional and Anatomical Connectivity Using Magnetic Resonance Imaging," Neuroimage, Vol. 16, No. 1, 2002, pp. 241-250.

http://dx.doi.org/10.1006/nimg.2001.1052

[16] P. Hagmann, J. P. Thiran, L. Jonasson, P. Vandergheynst, S. Clarke, P. Maeder, et al., "DTI Mapping of Human Brain Connectivity: Statistical Fibre Tracking and Virtual Dissection," Neuroimage, Vol. 19, No. 3, 2003, pp. 545554. http://dx.doi.org/10.1016/S1053-8119(03)00142-3

[17] A. E. Baird and S. Warach, "Magnetic Resonance Imaging of Acute Stroke," Journal of Cerebral Blood Flow \& Metabolism, Vol. 18, 1998, pp. 583-609. http://dx.doi.org/10.1097/00004647-199806000-00001

[18] F. Calamante, D. L. Thomas, G. S. Pell, J. Wiersma and R. Turner, "Measuring Cerebral Blood Flow Using Magnetic Resonance Imaging Techniques," Journal of Cerebral Blood Flow \& Metabolism, Vol. 19, 1999, pp. 701735. http://dx.doi.org/10.1097/00004647-199907000-00001

[19] M. A. Aweda, M. Agida, M. Dada, O. B. Awojoyogbe, K. Isah, O. P. Faromika, K. Boubaker, K. De and O. S. Ojambati, "A Solution to Laser-Induced Heat Equation inside a Two-Layer Tissue Model Using Boubaker Polynomials Expansion Scheme," Journal of Laser Micro/Nanoengineering, Vol. 6, No. 2, 2011, pp. 105-109.

[20] M. A. Aweda, M. Agida, M. Dada, O. B. Awojoyogbe, K. Isah, O. P. Faromika, K. Boubaker, K. De and O. S. Ojambati, "Boubaker Polynomials Expansion Scheme Solution to the Heat Transfer Equation inside Laser Heated
Biological Tissues," Journal of Heat Transfer, Vol. 134, No. 6, 2012, Article ID: 064503.

http://dx.doi.org/10.1115/1.4005744

[21] A. Belhadj, O. Onyango and N. Rozibaeva, "Boubaker Polynomials Expansion Scheme-Related Heat Transfer Investigation inside Keyhole Model," Journal of Thermophysics and Heat Transfer, Vol. 23, No. 3, 2009, pp. 639-642. http://dx.doi.org/10.2514/1.41850

[22] A. S. Kumar, "An Analytical Solution to Applied Mathematics-Related Love's Equation Using the Boubaker Polynomials Expansion Scheme," Journal of the Franklin Institute, Vol. 347, No. 9, 2010, pp. 1755-1761. http://dx.doi.org/10.1016/j.jfranklin.2010.08.008

[23] A. Belhadj, J. Bessrour, M. Bouhafs and L. Barrallier, "Experimental and Theoretical Cooling Velocity Profile inside Laser Welded Metals Using Keyhole Approximation and Boubaker Polynomials Expansion," Journal of Thermal Analysis and Calorimetry, Vol. 97, No. 3, 2009, pp. 911-920. http://dx.doi.org/10.1007/s10973-009-0094-4

[24] P. Barry and A. Hennessy, "Meixner-Type Results for Riordan Arrays and Associated Integer Sequences, Section 6: The Boubaker Polynomials," Journal of Integer Sequences, Vol. 13, 2010, pp. 1-34.

[25] M. Agida and A. S. Kumar, "A Boubaker Polynomials Expansion Scheme Solution to Random Love Equation in the Case of a Rational Kernel, El," Journal of Theoretical Physics, Vol. 7, 2010, pp. 319-326.

[26] A. Yildirim, S. T. Mohyud-Din and D. H. Zhang, "Analytical Solutions to the Pulsed Klein-Gordon Equation Using Modified Variational Iteration Method (MVIM) and Boubaker Polynomials Expansion Scheme (BPES)," Computers and Mathematics with Applications, Vol. 59, No. 8, 2010, pp. 2473-2477.

http://dx.doi.org/10.1016/j.camwa.2009.12.026

[27] J. Ghanouchi, H. Labiadh and K. Boubaker, "An Attempt to Solve the Heat Transfer Equation in a Model of Pyrolysis Spray Using 4q-Order m-Boubaker Polynomials," International Journal of Heat and Technology, Vol. 26, 2008, pp. 49-53.

[28] O. B. Awojoyogbe and K. Boubaker, "A Solution to Bloch NMR Flow Equations for the Analysis of Hemodynamic Functions of Blood Flow System Using mBoubaker Polynomials," Current Applied Physics, Vol. 9, No. 1, 2009, pp. 278-288.

http://dx.doi.org/10.1016/j.cap.2008.01.019

[29] S. Slama, J. Bessrour, M. Bouhafs and K. B. Ben Mahmoud, "Numerical Distribution of Temperature as a Guide to Investigation of Melting Point Maximal Front Spatial Evolution During Resistance Spot Welding Using Boubaker Polynomials," Numerical Heat Transfer, Part $A$, Vol. 55, No. 4, 2009, pp. 401-404. http://dx.doi.org/10.1080/10407780902720783

[30] S. Slama, M. Bouhafs and K. B. Ben Mahmoud, "A Boubaker Polynomials Solution to Heat Equation for Monitoring A3 Point Evolution during Resistance Spot Welding," International Journal of Heat and Technology, Vol. 26, No. 2, 2008, pp. 141-146.

[31] S. Tabatabaei, T. Zhao, O. Awojoyogbe and F. Moses, 
"Cut-off Cooling Velocity Profiling inside a Keyhole Model Using the Boubaker Polynomials Expansion Scheme," International Journal of Heat and Mass Transfer, Vol. 45, No. 10, 2009, pp. 1247-1255.

http://dx.doi.org/10.1007/s00231-009-0493-x

[32] S. Fridjine and M. Amlouk, "A New Parameter: An ABACUS for Optimizing Functional Materials Using the Boubaker Polynomials Expansion Scheme," Modern Physics Letters B, Vol. 23, No. 17, 2009, pp. 2179-2182. http://dx.doi.org/10.1142/S0217984909020321

[33] A. Milgram, "The Stability of the Boubaker Polynomials Expansion Scheme (BPES)-Based Solution to Lotka-Volterra Problem," Journal of Theoretical Biology, Vol. 271,
No. 1, 2011, pp. 157-158.

http://dx.doi.org/10.1016/j.jtbi.2010.12.002

[34] O. B. Awojoyogbe, "Analytical Solution of the Time Dependent Bloch NMR Equations: A Translational Mechanical Approach," Physica A, Vol. 339, No. 3-4, 2004, pp. 437-460. http://dx.doi.org/10.1016/j.physa.2004.03.061

[35] O. B. Awojoyogbe, O. M. Dada, O. P. Faromika and O. E. Dada, "Mathematical Concept of the Bloch Flow Equations for General Magnetic Resonance Imaging: A Review," Concepts in Magnetic Resonance Part A, Vol. 38A, No. 3, 2011, pp. 85-101. http://dx.doi.org/10.1002/cmr.a.20210 